\title{
Direct Observations of Photoexcitation Induced Dynamics of Charge Density Wave and Charge-Orbital Ordered State Using 2.8 MeV Ultrafast Electron Diffraction
}

\author{
Yimei Zhu ${ }^{1}$, P. Zhu ${ }^{1,2}$, L.Wu ${ }^{1}$, J. Li ${ }^{1}$, T. Konstantinova ${ }^{1}$, J. Cao $^{3}$, Y. Hidaka ${ }^{1}$, J.P. Hill ${ }^{1}$ and X.J. Wang ${ }^{1,4}$ \\ 1. Brookhaven National Laboratory, Upton, NY 11973 USA \\ 2. Shanghai Jiao Tong University, Shanghai 200240 China \\ ${ }^{3}$ Florida State University, Tallahassee, FL 32310 USA \\ ${ }^{4}$ SLAC National Accelerator Laboratory, Menlo Park, CA 94025 USA
}

The recent development of ultrafast imaging and diffraction opened a new frontier for studying structural dynamics at nanoscales. It has become one of the key directions for future electron microscopy. Ultrafast electron microscopy combines the superior spatial resolution of conventional electron microscope with short electron pulses enabling the detection of electronic and atomic motion on their natural time and lengths scales. Two paths have been pursued by modifying commercial TEMs. One is the multi-shot UEM [1] utilizing the single electron limit (one or few electrons per pulse) that circumvents the repulsive space charge problem but requires multimillion-shot accumulation to get a decent image thus suitable for repeatable dynamic events. The other is the single-shot DTEM [2] to achieve nm- $\mu$ s resolution. In parallel home-built ultrafast electron diffraction (UED) instruments have also been developed, especially the RF-based UED using accelerator technology that enabled to developing x-ray free electron lasers. Among the state-of-the-art electron sources, only photocathode RF guns are capable of generating $>10^{7}$ electrons per pulse, multi-MeV energy and sub ps-long electron beams. The distinct merit of photocathode RF guns is the very high acceleration gradient $(>100 \mathrm{MV} / \mathrm{m})$, over two-order of magnitude higher than $50 \mathrm{KeV}$ DC-guns [3]. It enables to minimize considerably the space charge effects, which can be scaled as inversely proportion to the product of velocity square and energy cube of the electrons, thus yielding much higher current density and beam brightness as recently demonstrated at Brookhaven $[4,5]$.

Here we present two case studies on photoinduced structural dynamics of the charge-density wave (CDW) state in $2 \mathrm{H}-\mathrm{TaSe}_{2}$ [4] and the charge-orbital ordered state in bi-layered $\mathrm{LaSr}_{2} \mathrm{Mn}_{2} \mathrm{O}_{7}$ at $77 \mathrm{~K}$ using $\mathrm{MeV}-\mathrm{UED}$. By simultaneously tracking both the melting of the periodic lattice distortion (PLD) associated with the CDW and the lattice heating, following an impulsive photoexcitation, the separate contributions of electronic excitation and lattice thermalization to the melting process are disentangled in the time domain. Distinct time-constants, reflecting the corresponding individual dynamics of the electronic and lattice systems, are observed. Our results demonstrate, for the first time in $2 \mathrm{H}-\mathrm{TaSe}_{2}$, that the PLD is first suppressed promptly by the electronic excitation and scattering, and then subsequently by lattice thermalization through electron-phonon interaction, on a much longer time scale, which leads to the final, full melting of the PLD. For $\mathrm{LaSr}_{2} \mathrm{Mn}_{2} \mathrm{O}_{7}$ our time-resolved electron diffraction study reveals the dynamic path of atoms and ions and indicates that the relative intensity change before and after the photoexcitation of the charge-order $(\mathrm{CO})$ superlattice peaks mostly results from the orbital order (OO). Among various phonon modes Jahn-Teller distortion dominates the OO process and the CO plays a minimal role during the electronic excitation. Our study shed light on the various and often intertwined degrees of freedom and their responses to external perturbations in strongly correlated electron systems [6].

\section{References:}

[1] D.J. Flannigan et al, PNAS, 1079933 (2010).

[2] J.S. Kim et al, Science 3211472 (2008).

[3] M. Eichberger et al, Nature 468, 799 (2010)

[4] P. Zhu et al, Appl. Phys. Lett., 103071914 (2013).

[5] P. Zhu et al, "Femtosecond Time-resolved MeV Electron Diffraction", submitted.

[6] The authors would like to thank S.W. Cheong, J. Geck, S. Pjerov, T. Ritschel, H.Berger, Y.Shen, and R.Tobey for providing samples and assistance. Work supported by the U.S. DOE, under contract No. DE-SC0012704. 


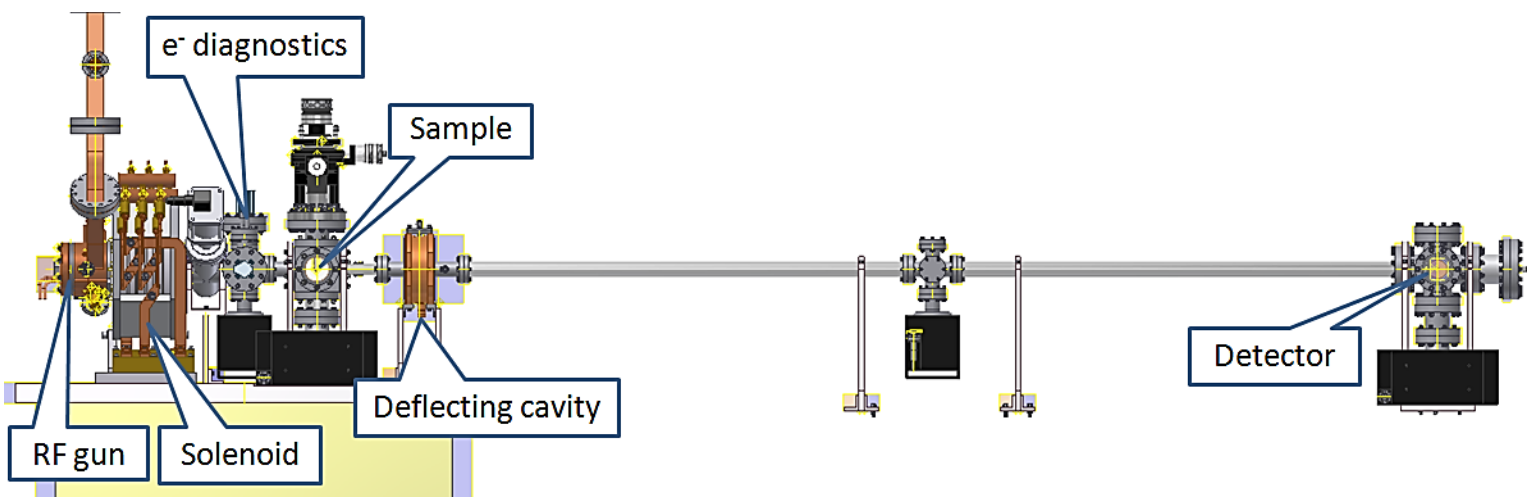

Fig. 1. Schematic of the relativistic MeV-UED (ultrafast electron diffraction) set-up at Brookhaven national Laboratory. The entire system is $\sim 4.5 \mathrm{~m}$ long. UV photons from a Ti-sapphire laser are used to generate electrons in the RF gun. A solenoid magnet focuses the beam onto the detector screen $4 \mathrm{~m}$ downstream of the sample. NearIR pulses from the same Ti-sapphire laser are used to optically pump the sample. The instrument has a cryogenic capability and can operates at $2-4 \mathrm{MeV}$ with $10^{6}$ electron per pulse to achieve $120 \mathrm{fs}$ temporal resolution.
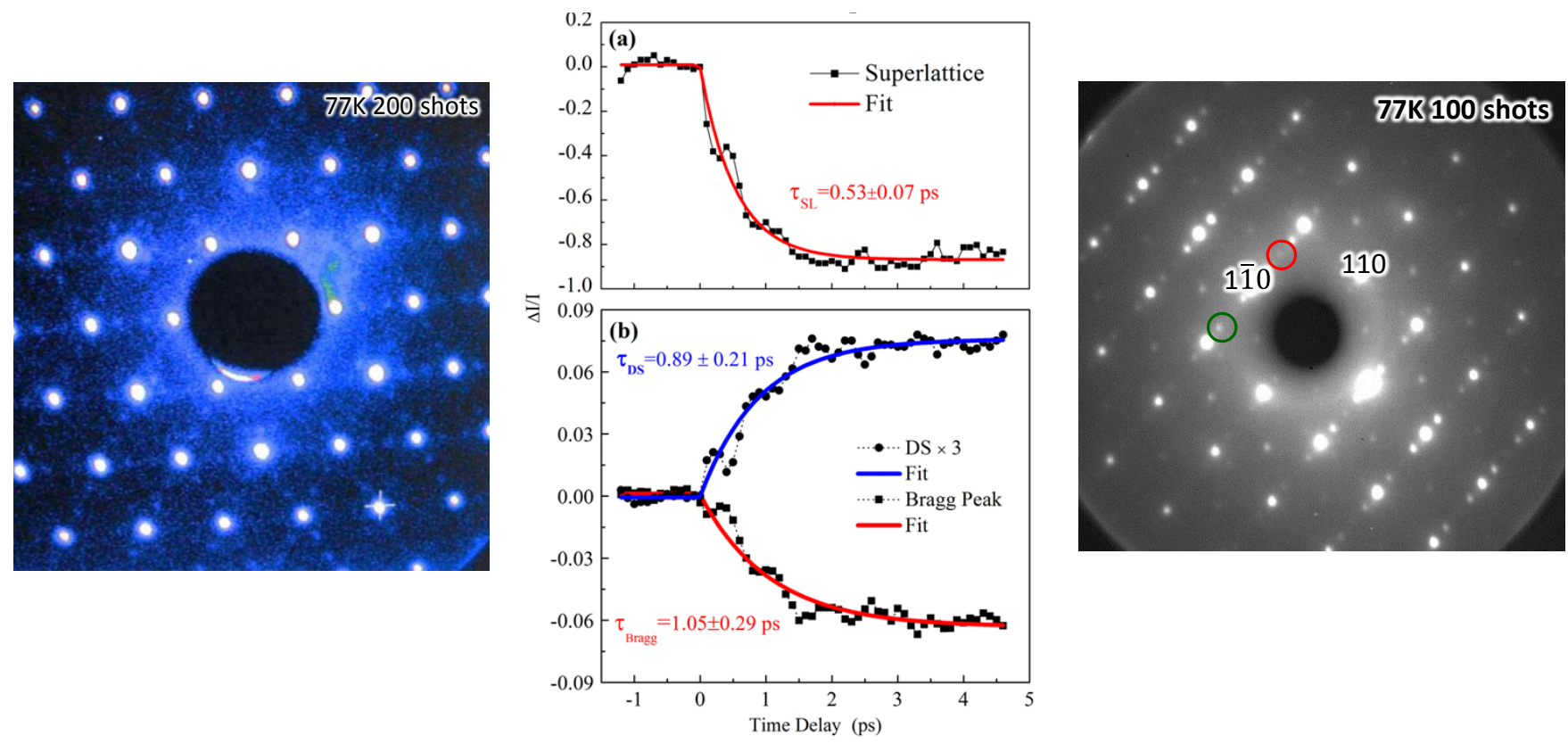

Fig. 2 Photoexcitation induced MeV UED diffraction data from: (left panel) Charge density wave (CDW) state in $2 \mathrm{H}-\mathrm{TaSe}_{2}$; (right panel) Charge ordered state (marked by the red circle) and the orbital ordered state (marked by the green circle) in $\mathrm{LaSr}_{2} \mathrm{Mn}_{2} \mathrm{O}_{7}$. (center panel): Temporal evolution of the CDW peak (a) and the Bragg peak and diffuse scattering intensity (b) in $2 \mathrm{H}-\mathrm{TaSe}_{2}$. For clarity, the change of diffuse scattering intensity is multiplied by 3 . The pump was a $795 \mathrm{~nm}$ optical pulse at a fluence of $1.4 \mathrm{~mJ} / \mathrm{cm}^{2}$. The solid lines with given time constants are fits to the experimental data using an exponential function. 